\title{
Microstructure refinement mechanism of undercooled $\mathrm{Cu}_{55} \mathrm{Ni}_{45}$ alloys
}

\author{
Hongfu Wang ${ }^{1, *}$, Cheng Tang ${ }^{1}$, Hongen $\mathrm{An}^{2}$, Yuhong Zhao ${ }^{3, \dagger}$ \\ ${ }^{1}$ School of Mechanical Engineering, North University of China, Taiyuan, Shanxi 030051, China \\ ${ }^{2}$ Faculty of Engineering, University Malaysia Sabah, Kota Kinabalu 88400, Malaysia \\ ${ }^{3}$ College of Materials Science and Engineering, North University of China, Taiyuan, Shanxi 030051, China
}

\begin{abstract}
Different undercooling degrees of $\mathrm{Cu}_{55} \mathrm{Ni}_{45}$ alloy were obtained by the combination of molten glass purification and cyclic superheating, and the maximum undercooling degree reached $284 \mathrm{~K}$. The microstructure of the alloy was observed by metallographic microscope, and the evolution of microstructure was studied systematically. There are two occasions of grain refinement in the solidification structure of the alloy: one occurs in the case of low undercooling, and the other occurs in the case of high undercooling. Electron backscatter diffraction (EBSD) technology was used to analyze the rapid solidification structure under high undercooling. The features of flat polygonal grain boundary, high proportion of twin boundary, and large proportion of large angle grain boundary indicate recrystallization. The change in microhardness of the alloy under different undercooling degrees was studied by microhardness tester. It was found that the average microhardness decreased sharply at high undercooling degrees, which further confirmed the recrystallization of the solidified structure at high undercooling degrees.
\end{abstract}

Keywords: solidification structure, rapid solidification, grain refinement, recrystallization

\section{Introduction}

The properties of materials are related not only to the composition but also closely to the existing form of structure. Because of its excellent mechanical properties, materials with fine and uniform grain structure are often directly cast into engineering application components or used as initial raw materials for further machining. Therefore, the means to make the material grain refinement and study the mechanism of grain refinement has been the focus of research [1-3].

There are many techniques for grain refinement, one of which is deep undercooling rapid solidification [4]. The deep undercooling technology includes the deep undercooling of bulk metals, and examples of this technology include the molten glass purification method [5], the circulation superheating method [6], and the combination of molten glass purification and circulation superheating method [7]. Another technique is the

\footnotetext{
* E-mail: wanghongfu@nuc.edu.cn

† E-mail: zhaoyuhong@nuc.edu.cn
}

deep undercooling of small metal droplets, and examples of this technology include the emulsification thermal analysis method [8], the falling tube method [9], and the containerless electromagnetic suspension melting method [10]. It is generally believed that only the undercooling degree of alloy or metal melt is required; the grain refinement will occur in the rapid solidification process and subsequent solidification stage.

To study the relationship between grain refinement mechanism and undercooling, scholars put forward the following theories to explain the grain refinement mechanism: (1) The dynamic nucleation mechanism proposed by Horvey [11] is mainly confirmed in pure nickel and some nickel base alloys. (2) Critical velocity theory, proposed by Willnecker [12]. (3) Recrystallization mechanism [13, 14]. (4) Dendrite remelting and fracture mechanism, etc. The dendrite remelting effect includes two aspects; one is driven by solid-liquid interfacial tension proposed by Karma [15]; the other is caused by chemical superheating proposed by $\mathrm{Li}$ [16]. It is generally believed that the grain re- 
finement under low undercooling is caused by dendrite remelting and fracture in the process of recalescence and subsequent stages. However, there is no unified view on the causes of grain refinement under large undercooling, and there are still many disputes. Recrystallization mechanism is one of the mechanisms that may refine the grains under high undercooling. More and more people begin to verify it from the experimental point of view [17-19]. The recrystallization mechanism suggests that the recrystallization is induced by stress [20]. When the undercooling degree of undercooled melt is greater than the critical undercooling degree, the proportion of primary solid phase formed in the early stage of rapid solidification will continue to increase and a continuous dendrite network will be formed. Solidification shrinkage and thermal strain induced liquid flow between dendrites will lead to stress accumulation in the primary dendrite skeleton. With the increase of undercooling, when the accumulated stress exceeds the strength limit of the alloy, the primary dendrite skeleton will undergo plastic deformation and stress fracture. The broken dendrite fragments store strain energy and provide driving force for subsequent recrystallization, which makes the grains refined into fine and uniform equiaxed grains.

In this paper, the microstructural evolution of $\mathrm{Cu}_{55} \mathrm{Ni}_{45}$ alloy has been systematically studied by means of the combination of molten glass purification and cyclic superheating. Two grain refinement phenomena have been found under low undercooling and high undercooling. The electron backscatter diffraction (EBSD) technique was used to characterize the grain refinement structure. The hardness test of the alloy showed that the sharp decrease of microhardness under high undercooling was the indirect evidence of recrystallization.

\section{Experimental steps}

Pure copper (99.99\%) and pure nickel (99.99\%) weighed in a certain proportion are smelted in-situ in a vacuum arc melting furnace under an argon atmosphere to prepare $\mathrm{Cu}_{55} \mathrm{Ni}_{45}$ master alloy. The alloy was remelted at least three times to ensure that it was well-mixed, and $4 \mathrm{~g}$ sample was cut from each original ingot for experiments. The alloy sample and quartz tube were cleaned in the ultrasonic cleaner for $30 \mathrm{~min}$ to remove the surface impurities. After the alloy sample and quartz tube were dried, the sample was put into the tube, and the treated $\mathrm{B}_{2} \mathrm{O}_{3}$ purification agent was added. Finally, the quartz tube was put into the high frequency induction coil, the vacuum of the experimental furnace was pumped to $3 \times 10^{-3} \mathrm{~Pa}$, and argon was backfilled to $5 \times 10^{-2} \mathrm{~Pa}$. An infrared thermometer with a response time of $1 \mathrm{~ms}$ was used to record the temperature curve during the whole solidification process. First, the temperature was slowly raised to $750^{\circ} \mathrm{C}$ for $15 \mathrm{~min}$ to melt $\mathrm{B}_{2} \mathrm{O}_{3}$ and fully cover the alloy. Second, when the temperature was higher than the melting point of the alloy from $100 \mathrm{~K}$ to $200 \mathrm{~K}$ and kept for $15 \mathrm{~min}, \mathrm{~B}_{2} \mathrm{O}_{3}$ purifier could fully absorb impurities to inhibit heterogeneous nucleation. And finally, the cyclic superheating method is used until the ideal degree of undercooling is obtained. The experimental diagram is shown in Figure 1.

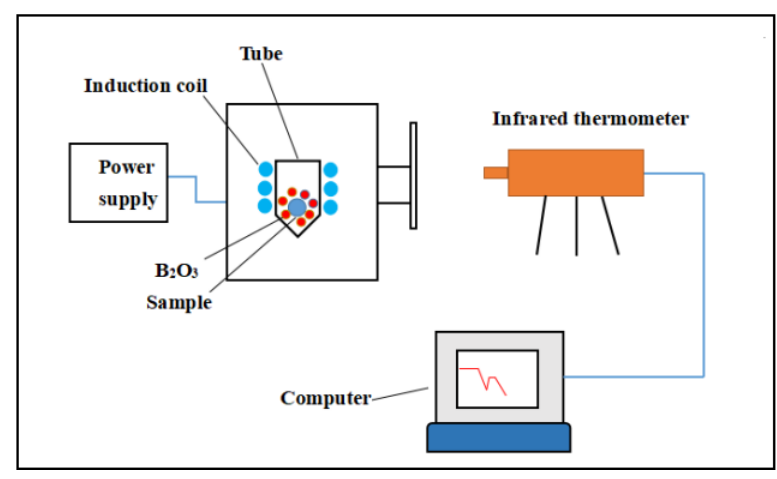

Fig. 1. Schematic diagram of undercooling experimental device.

After cutting, inlaying, and polishing, the cooled samples were etched with $20 \mathrm{ml} \mathrm{HCl}$ and $20 \mathrm{ml} \mathrm{HNO}_{3}$ mixed acid solution. Then, the morphology of undercooled microstructure was observed by metallographic microscope (OM, Leica DM2500M) and the pictures were taken. To observe the grain and grain boundary characteristics of the samples, the samples were polished with $\mathrm{Al}_{2} \mathrm{O}_{3}$ colloidal suspension for $10 \mathrm{~h}$ on a vibration polishing machine, and then the samples were detected by EBSD. Finally, the microhardness of 
all samples was measured by microhardness tester (HMV-2T), and the load was $490.3 \mathrm{mN}$ for $15 \mathrm{~s}$. It was ensured that at least 40 points were tested for each sample, and also that these were evenly distributed on the whole sample surface in a cross shape; and the average value was taken as the final hardness value.

\section{Results and discussion}

\subsection{Microstructure analysis}

Different undercooling degrees of $\mathrm{Cu}_{55} \mathrm{Ni}_{45}$ alloy were obtained by the combination of molten glass purification and cyclic superheating, and the temperature curve of alloy undercooling experiment was recorded by infrared thermometer. A sample was obtained every $10 \mathrm{~K}$ undercooling, and finally lots of samples with an undercooling range of $47 \mathrm{~K}-284 \mathrm{~K}$ were obtained. In the whole range of undercooling, the following microstructure transformation occurs:

1. $0 \mathrm{~K}<\Delta T<54 \mathrm{~K}$. When the undercooling is lower than $54 \mathrm{~K}, \Delta T=47 \mathrm{~K}$. As can be seen from Figure 2A, the solidification microstructure of the alloy shows coarse dendrite with large dendrite size, and welldeveloped secondary dendrite arm fully surrounding the main dendrite without obvious specific growth direction. According to the BCT [21] model, in the undercooling range, the dendrite growth is controlled by solute diffusion, and the alloy is confined in a very narrow region around it, thus forming coarse dendrite.

2. $54 \mathrm{~K} \leq \Delta T<96 \mathrm{~K}$. In the undercooling range, with the increase of undercooling degree, the proportion of coarse dendrites decreases, and the microstructure morphology changes into relatively small dendrites. And the proportion of quasi-spherical equiaxed crystals continues to increase, as shown in the lower right corner of Figure 2B. When $\Delta T=70 \mathrm{~K}$, most grains are refined into spherical equiaxed grains, and the first grain refinement occurs, as shown in Figure 2C. With the further increase of undercooling, some directional dendrites appear in the microstructure; and the reason is that in the overcooling range, the dendrite growth factor controlled by thermal diffusion increases gradually, and the dendrite grows in the direction of thermal diffusion, thus showing directional characteristics, as shown in Figure $2 \mathrm{D}$. The whole dendrite growth process is affected by solute diffusion and thermal diffusion.

3. $96 \mathrm{~K} \leq \Delta T<227 \mathrm{~K}$. In the undercooling range, with the increase of undercooling, the first refined grain disappears, and the microstructure presents fine dendrite with very small dendrite spacing and strong orientation. According to the BCT model, in the undercooling range, the influence of solute diffusion is very low, and the growth factor of dendrite controlled by thermal diffusion is dominant. The growth range controlled by thermal diffusion is much larger than that controlled by solute diffusion, so the microstructure shows the development of extremely fine dendrite network, as shown in Figure 2E.

4. $\Delta T \geq 227 \mathrm{~K}$. When the undercooling exceeds $227 \mathrm{~K}$, the fine dendrite dominated by thermal diffusion breaks, equiaxed grains appear, and the second grain refinement occurs; $\Delta T=227 \mathrm{~K}$, as shown in Figure $2 \mathrm{~F}$. The first grain refinement and the second grain refinement differ noticeably. The microstructure is characterized by a large number of polygonal equiaxed grains, flat grain boundaries, and annealing twins, as shown in Figure 2G, 2H. These are the typical characteristics of recrystallization of solidified structure under large undercooling, indicating that the undercooled melt recrystallized in the later stage of rapid solidification.

Through the analysis of microstructure in the whole undercooling range (47-284 K), we found that $\mathrm{Cu}_{55} \mathrm{Ni}_{45}$ experienced the evolution process of "coarse dendrite - equiaxed crystal - oriented fine dendrite - equiaxed crystal," the grain refinement occurred at low and high undercooling, and 

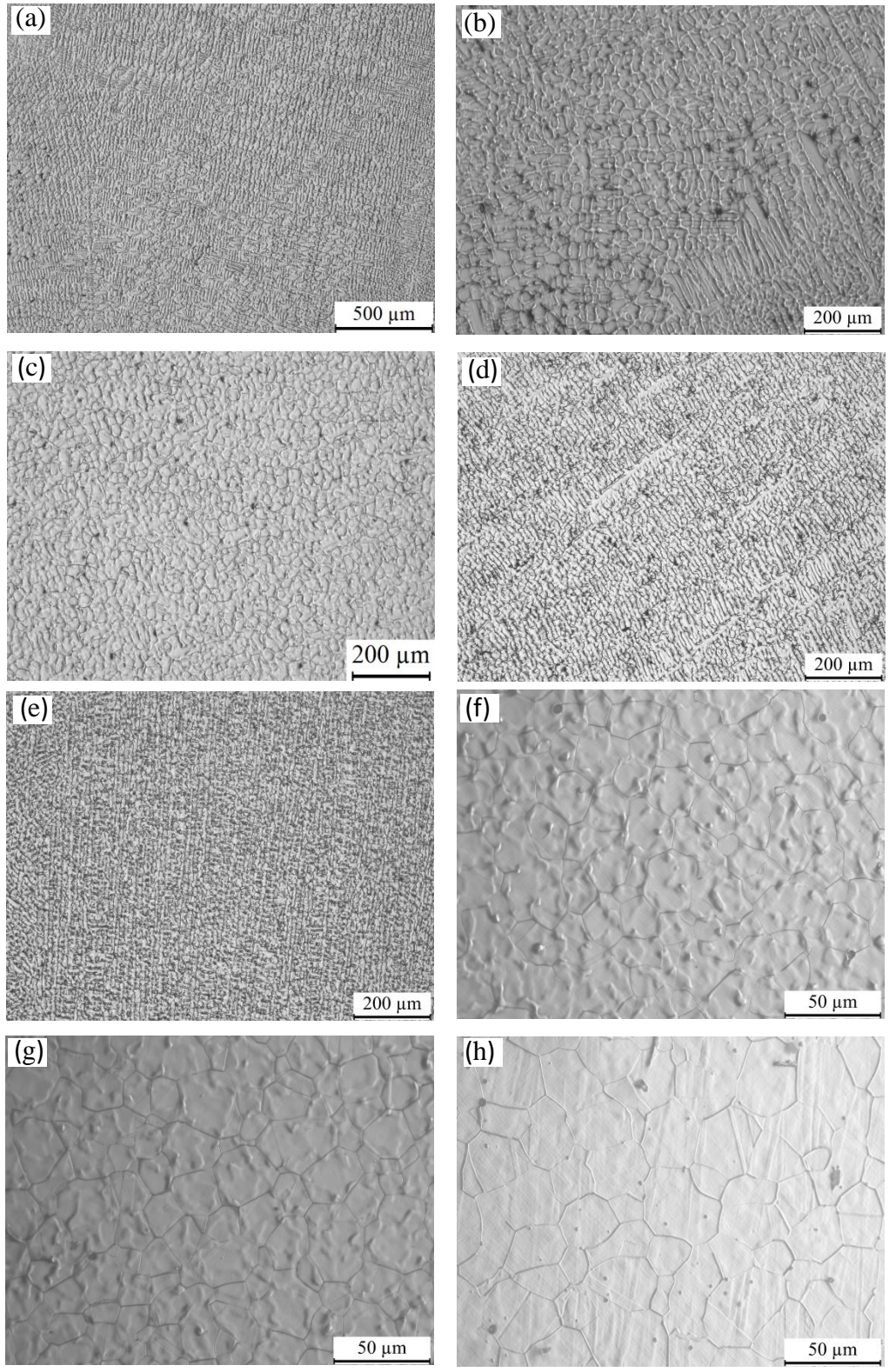

Fig. 2. Microstructure of $\mathrm{Cu}_{55} \mathrm{Ni}_{45}$ alloy under different undercooling degrees. [(A) $\Delta T=47 \mathrm{~K}$, (B) $\Delta T=68 \mathrm{~K}$, (C) $\Delta T=70 \mathrm{~K}$, (D) $\Delta T=91 \mathrm{~K}$, (E) $\Delta T=187 \mathrm{~K}$, (F) $\Delta T=227 \mathrm{~K}$, (G) $\Delta T=272 \mathrm{~K}$, (H) $\Delta T=284 \mathrm{~K}]$.

the morphology of the two grain refinement structures was very different. Based on the analysis of the high undercooling of experimental samples, the microstructure with undercooling of $227 \mathrm{~K}, 274 \mathrm{~K}$, and $284 \mathrm{~K}$ are polygonal equiaxed crystals, and there are a large number of annealing twins and a high proportion of large angle grain boundaries. It can be inferred that the microstructure with undercooling $>284 \mathrm{~K}$ also has the same microstructure characteristics as above due to the second grain refinement.

The BCT [21] model can describe the dendrite precipitation process in undercooled melt and the evolution of four undercooling components with initial undercooling degree. The dendrite tip undercooling degree $(\Delta T)$ consists of four undercooling components: thermal undercooling degree $\left(\Delta T_{t}\right)$, solute undercooling degree $\left(\Delta T_{c}\right)$, curvature under- 
cooling degree $\left(\Delta T_{r}\right)$, and dynamic undercooling degree $\left(\Delta T_{k}\right)$ :

$$
\Delta T=\Delta T_{t}+\Delta T_{c}+\Delta T_{r}+\Delta T_{k}
$$

In the evolution process of microstructure morphology of $\mathrm{Cu}_{55} \mathrm{Ni}_{45}$ alloy: when undercooling $\Delta T<54 \mathrm{~K}$, solute undercooling $\Delta T_{c}$ dominates the dendrite precipitation process and forms coarse dendrite (Figure 2A). When the undercooling is in the range of $54 \mathrm{~K} \leq \Delta T<96 \mathrm{~K}$, the effect of thermal undercooling $\Delta T_{t}$ begins to increase, showing a small proportion of directional dendrites (Figure 2D), and the first grain refinement occurs at $\Delta T=70 \mathrm{~K}$. With the gradual increase of the degree of undercooling, the degree of thermal undercooling $\Delta T_{t}$ increases significantly. The dendrites grow in the direction of thermal diffusion, the orientation is gradually enhanced, and the dendrites are getting finer (Figure 2E). The degree of dynamic undercooling $\Delta T_{k}$ also begins to increase. When undercooling $\Delta T$ is greater than $227 \mathrm{~K}$, the second grain refinement occurs. Since the solute interception effect [22] is more obvious when the undercooling is high, the solute atoms are wrapped in the solid phase by the rapidly advancing interface before diffusion. At this time, the dynamic undercooling $\Delta T_{k}$ mainly caused by interface factors $[23,24]$ will exceed the thermal undercooling $\Delta T_{t}$. The dendrite or grain size of all alloy samples during solidification is relatively large (Figure 8A), so the curvature undercooling $\Delta T_{r}$ has little effect on the interface morphology. Therefore, the influence of curvature undercooling $\Delta T_{r}$ is always low.

\subsection{Grain refinement mechanism}

\subsubsection{Grain refinement mechanism under low undercooling}

The solidification structure of $\mathrm{Cu}_{55} \mathrm{Ni}_{45}$ alloy undergoes first grain refinement at the undercooling degree of 54-96 K, and complete refinement occurs at $\Delta T=70 \mathrm{~K}$. The dendrite remelting fracture caused by chemical superheating proposed by Li [16] can clearly explain the grain refinement mechanism under low undercooling. The mechanism of dendrite remelting and fracture points out that when the undercooled melt temperature is lower than a certain temperature of the equilibrium liquidus, a large amount of solidification latent heat will be released. The release of latent heat causes the temperature of the system to rise rapidly beyond the solidus temperature, which causes the primary dendrite skeleton to superheat and remelt, and finally break into fine equiaxed crystals. According to the calculation of the remelting ratio of $\mathrm{Li}$ [16] to the dendrite of $\mathrm{Ni}_{75} \mathrm{Cu}_{25}$ alloy, the highest remelting ratio of about $90 \%$ can be obtained at undercooling $\Delta T=40 \mathrm{~K}$, and the corresponding microstructure morphology is mostly fine equiaxed grains. As the undercooling $\Delta T$ increases within a certain range, the proportion of remelting in the alloy continues to decrease, its microstructure presents equiaxed crystals and dendrites, and the proportion of dendrites waxes higher and higher. This study well confirms the mechanism of dendrites' fragmentation due to superheating and remelting at low undercooling.

Figure 3 shows several typical solidification recalescence curves of $\mathrm{Cu}_{55} \mathrm{Ni}_{45}$ alloy. With the increase of undercooling, the rapid solidification time becomes shorter and shorter, and the maximum recalescence temperature $T_{R}$ [25] decreases gradually. The maximum recalescence temperature $T_{R}$ of undercooling $\Delta T$ at $54 \mathrm{~K}, 70 \mathrm{~K}$, and $96 \mathrm{~K}$ is higher than that of equilibrium solidus, which also confirms the mechanism mentioned by Li [16], who proposed that the dendrites are broken due to superheating and remelting at low undercooling and that the grains are refined.

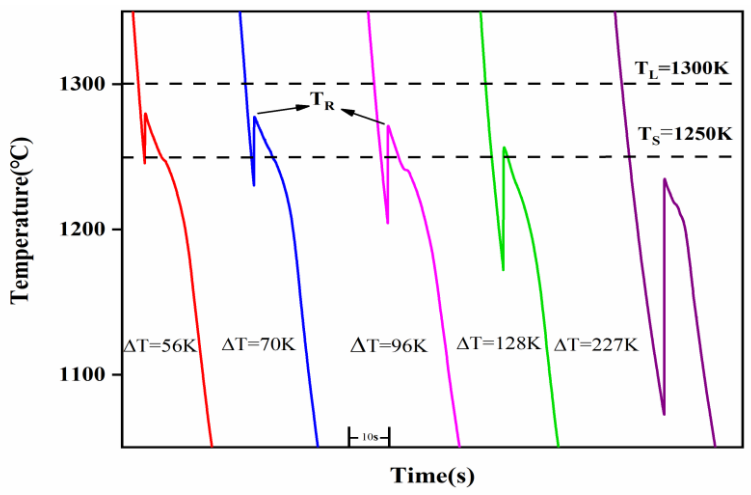

Fig. 3. Solidification recalescence curve of $\mathrm{Cu}_{55} \mathrm{Ni}_{45}$ alloy. 

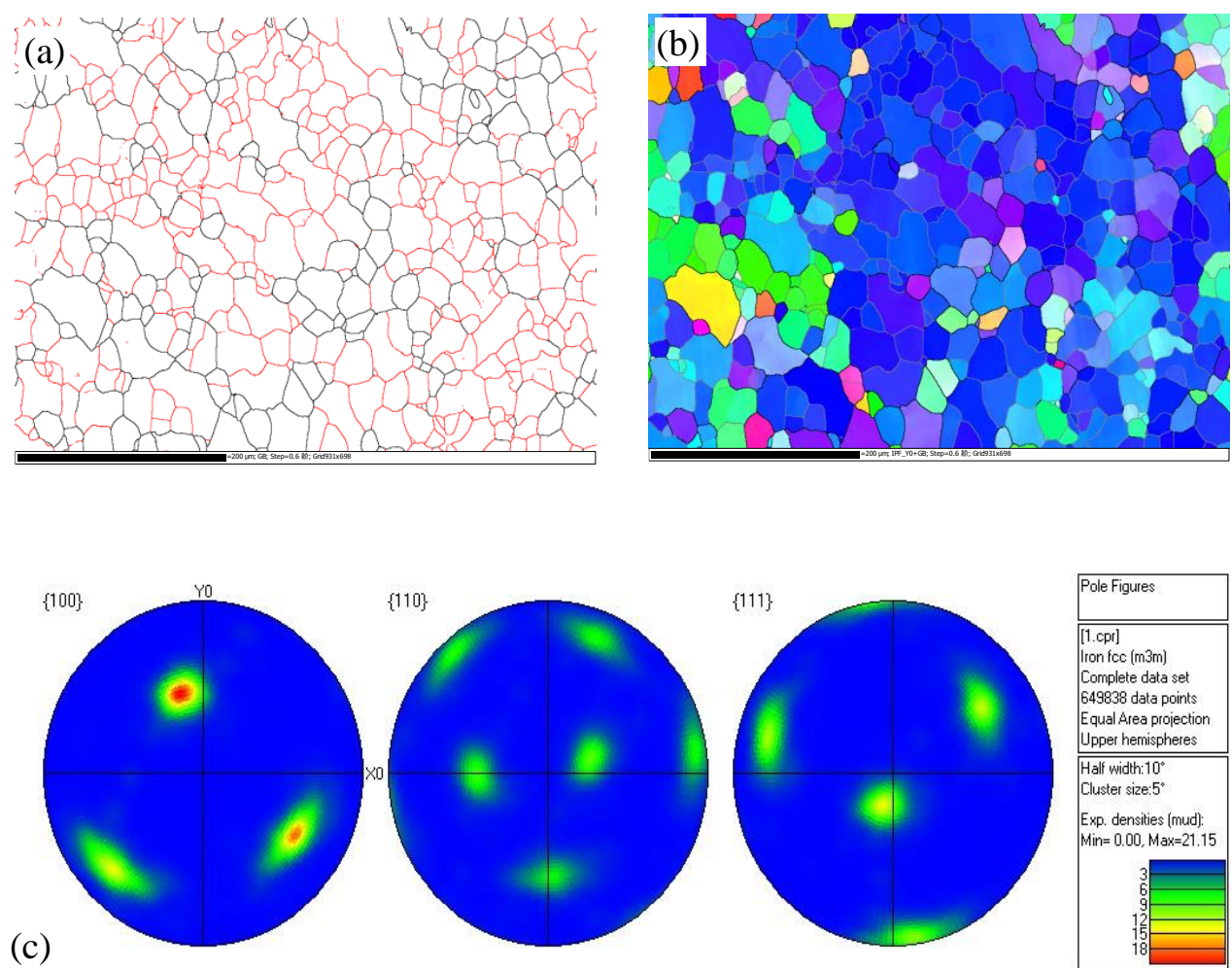

(d)

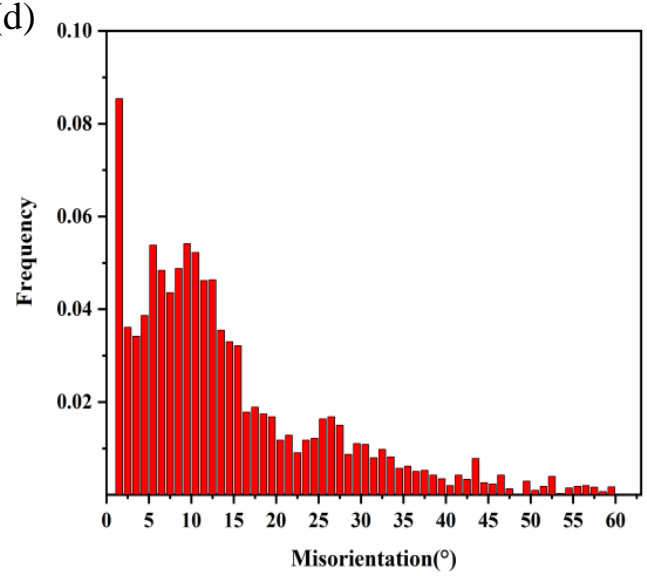

Fig. 4. EBSD characterization of $\mathrm{Cu}_{55} \mathrm{Ni}_{45}$ alloy with undercooling of $70 \mathrm{~K}$. (A) Grain boundary diagram of microstructure of $70 \mathrm{~K}$ undercooling alloy, $(\mathbf{B})$ grain boundary orientation diagram, $(\mathbf{C})$ pole diagram of $(\mathbf{B}, \mathbf{D})$. distribution diagram of grain boundary orientation difference of (B). EBSD, electron backscatter diffraction.

The grain refinement structure of $\mathrm{Cu}_{55} \mathrm{Ni}_{45}$ alloy with low undercooling $\Delta T=70 \mathrm{~K}$ was characterized by EBSD. The EBSD analysis diagram is shown in Figure 4.
Here, we consider the grain boundary orientation difference $<15^{\circ}$ as small angle grain boundary. The solidification microstructure of $\mathrm{Cu}_{55} \mathrm{Ni}_{45}$ alloy with undercooling of about $70 \mathrm{~K}$ shows spherical 
equiaxed grains, curved grain boundaries, and no annealing twins (Figure 2C). The red lines in Figure $4 \mathrm{~A}$ represent small angle grain boundaries, and a large number of small angle grain boundaries can be clearly seen. In Figure 4B, different colors indicate different grain orientations, and the same or similar colors indicate the same orientation. A large number of grains with the same or similar orientations can be observed, and high-strength textures can be observed in Figure 4C.

Figure 4D is obtained by statistical calculation of grain boundary orientation difference, in which the small angle grain boundary orientation difference is as high as $69 \%$. The mechanism of dendrite overheat remelting fracture proposed by $\mathrm{Li}$ [16] well explains the phenomenon of grain refinement at low undercooling. When the undercooled melt releases a large amount of latent heat of crystallization due to rapid solidification, the temperature of the system rises rapidly and exceeds the solidus temperature, resulting in the primary dendrite superheating remelting fracture, which refines into smaller equiaxed grains in the subsequent slow solidification stage.

The primary solid remelting fraction of alloy liquid during rapid solidification has a great relationship with undercooling [26]. In agreement with results presented in previous studies, Li [16] proposed a formula for remelting fraction of primary solid phase:

$$
f_{L}=\frac{k_{0}\left(T_{R}-T_{S}\right) / \Delta T_{0}}{1-\left(1-k_{0}\right)\left(T_{R}-T_{S}\right) / \Delta T_{0}}
$$

where $T_{R}$ is the highest recalescence temperature corresponding to the degree of undercooling $\Delta T, T s$ is the equilibrium solidus temperature corresponding to the initial solid of the Cs component, $\Delta T_{0}$ is the equilibrium crystallization temperature range corresponding to the initial solid of the Cs component, and $k_{0}$ is the equilibrium solute partition coefficient. According to the formula, the calculated results are shown in Figure 5 as dimensionless remelting fraction of $\mathrm{Ni}_{55} \mathrm{Cu}_{45}$ alloy under different undercooling degrees.

The calculated results are in good agreement with the experimental results. The maximum remelting fraction is about $90 \%$ at the un-

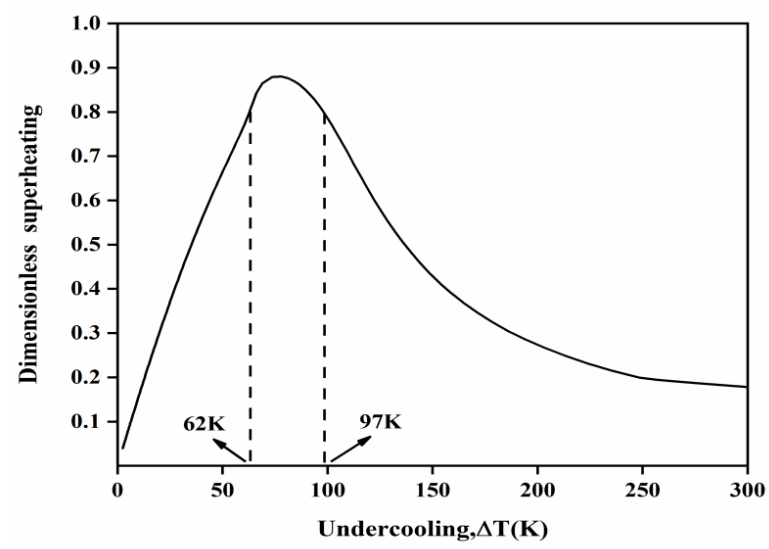

Fig. 5. The relationship between dimensionless dendrite remelting fraction and undercooling of $\mathrm{Cu}_{55} \mathrm{Ni}_{45}$ alloy.

dercooling of about $75 \mathrm{~K}$. The results show that the trend of dimensionless remelting fraction curve increases first and then decreases: the remelting fraction of $\mathrm{Cu}_{55} \mathrm{Ni}_{45}$ dendrite is very small at very low undercooling, and the corresponding microstructure is coarse dendrite. When the undercooling is $54-96 \mathrm{~K}$, the dendrite remelting fraction is at a relatively high value, and a large number of equiaxed grains and proportions and relatively small dendrites can be observed in the corresponding microstructure. At moderate undercooling, the remelting fraction decreases, and the microstructure of the alloy in the undercooling range of 128-227 K presents dendrite. With the increase of undercooling, the dendrite shows strong orientation and gradually decreasing dendrite spacing. At high undercooling of $227 \mathrm{~K}$, the remelting fraction has been significantly reduced, and the microstructure morphology is equiaxed grains with flat polygon. The proportion of remelted dendrites is very small, which is not enough to cause grain refinement. This shows that the grain refinement mechanism employed at high undercooling is a different one.

\subsubsection{Grain refinement mechanism under high undercooling}

Grain refinement occurs when undercooling of $\mathrm{Cu}_{55} \mathrm{Ni}_{45}$ alloy is greater than $227 \mathrm{~K}$. In contrast with the microstructure of the first grain refine- 

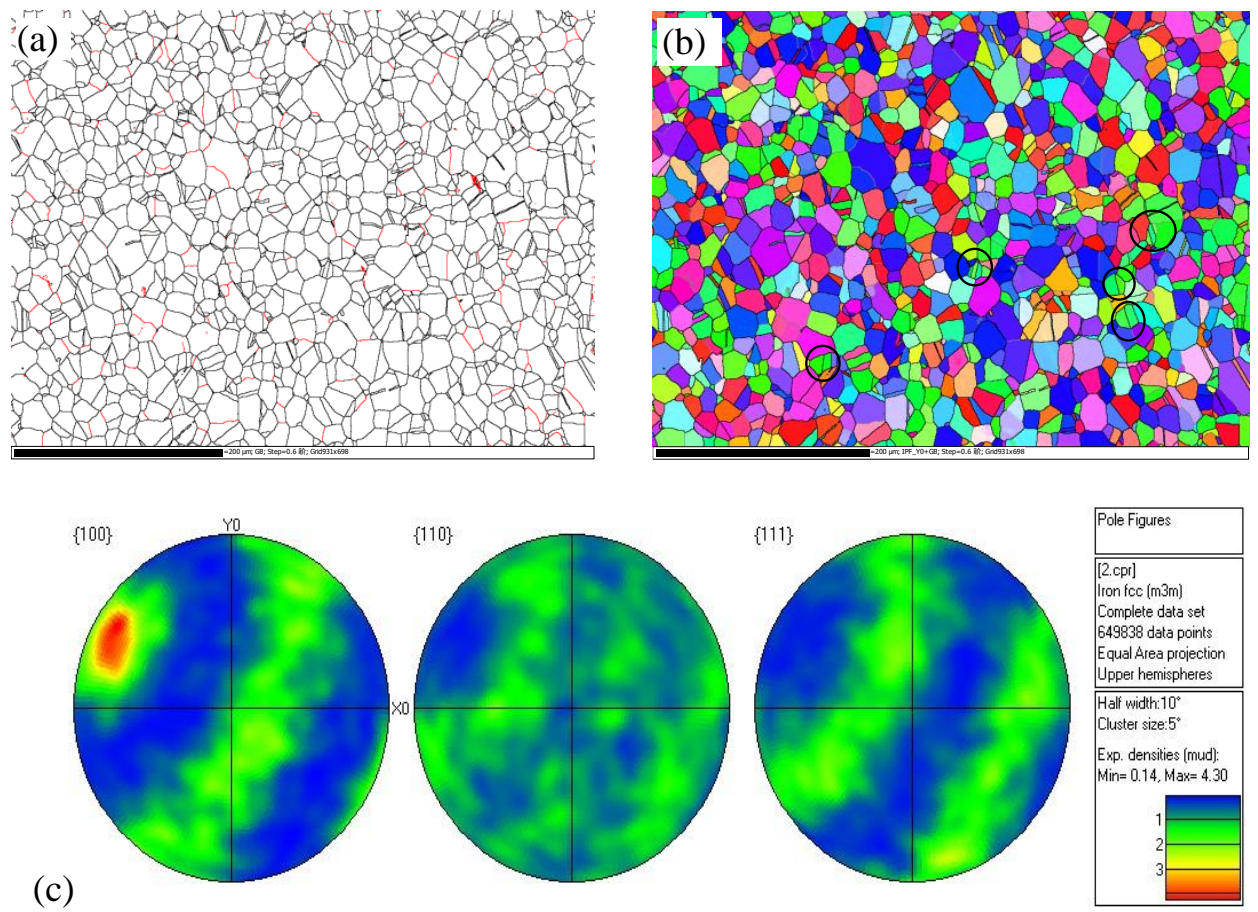

(d)

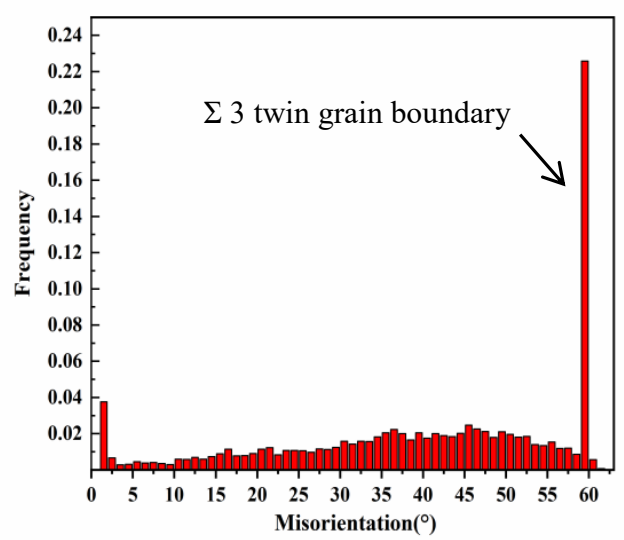

Fig. 6. EBSD characterization of $\mathrm{Cu}_{55} \mathrm{Ni}_{45}$ alloy with undercooling of $272 \mathrm{~K}$. (A) Grain boundary diagram of microstructure of $272 \mathrm{~K}$ undercooling alloy, (B) grain boundary orientation diagram, (C). pole diagram of (B, D). Distribution diagram of grain boundary orientation difference of (B). EBSD, electron backscatter diffraction.

ment, the second grain refinement has smaller grain size, flat polygonal grain boundary, and annealing twins - which are not found in the first grain refinement [17]. According to the remelting fraction calculated by the dendrite remelting formula, as shown in Figure 5, the proportion of dendrite remelting is very small under high undercooling, which is not enough to refine the grains. Stress induced recrystallization mechanism [20] is one of the potential mechanisms to refine the grains of the alloy. Stress induced recrystallization indicates that with the increase of the initial undercooling, the volume fraction of dendrite in the primary solid phase formed during rapid solidification will 
increase, and a continuous dendrite network will gradually form in the alloy liquid. Accordingly, the dendrite skeleton also begins to have strength, and then presents deformation resistance. When the undercooling degree of the undercooled melt is high, the accumulated stress will reach the strength limit of the alloy, and the dendrite framework will undergo plastic deformation, resulting in stress fracture. The broken dendrite fragments store the strain energy, which provides the driving force for the subsequent recrystallization behavior.

EBSD technology was used to characterize the grain refinement structure of $\mathrm{Cu}_{55} \mathrm{Ni}_{45}$ alloy with high undercooling $\Delta T=272 \mathrm{~K}$. The EBSD analysis diagram is shown in Figure 6. The black lines in Figure 6A represent large angle grain boundaries $\left(>15^{\circ}\right)$. It is evident that a large number of large angle grain boundaries can be seen.

The microstructure of $\Delta T=272 \mathrm{~K}$ undercooling $\mathrm{Cu}_{55} \mathrm{Ni}_{45}$ alloy is polygonal equiaxed grains with flat grain boundaries and a large number of annealing twins (shown in black circle in Figure 6B), which is a strong evidence of recrystallization of the alloy. In Figure 6B, different colors indicate different grain orientations, and the same or similar colors indicate the same orientation. The disordered colors indicate that the grain orientations are randomly distributed without obvious preferred orientation. No obvious high-strength texture was observed in the Figure 6C polar diagram. According to the statistical calculation of grain boundary orientation difference, it is shown in Figure 6D that the large angle grain boundary orientation difference is as high as $90 \%$, and the $\Sigma 3$ twin grain boundary $\left(<111>60^{\circ}\right)$ is as high as $22.5 \%$. The mechanism of dendrite superheat remelting mentioned above can not explain the EBSD characterization diagram of $\Delta T=272 \mathrm{~K}$ undercooling, but the mechanism of stress-induced recrystallization can explain it perfectly. With the increase of undercooling, the continuous dendrite network is formed gradually. In the rapid solidification stage, the dendritic framework produces deformation resistance due to thermal strain and the alloy liquid phase in the dendrite network region flows to the crystallization front. Once the accumulated stress exceeds the strength limit of the alloy, the dendrite frame- work will break, and the strain energy stored in the broken dendrite fragments will provide the driving force for the subsequent recrystallization. In the process of recrystallization, the recrystallized small angle grain boundaries will merge and migrate rapidly under the action of driving force, resulting in the grain boundary orientation difference becoming larger and larger, which will be gradually engulfed by large angle grain boundaries. The grain boundaries will gradually become flat from bending, and finally form large angle grains with flat grain boundaries. In addition, due to the accumulated stress in the primary dendrites, the dendrites are randomly distributed after breaking, and a small part of the remelted dendrites are also randomly distributed. Therefore, no new texture is introduced during recrystallization and grain growth, and the grain orientation is random [27].

Based on the solidification shrinkage and thermal shrinkage of undercooled melt during rapid solidification, Liu [20] proposed a stress accumulation model, which can semi-quantitatively estimate the stress accumulation in the solidification structure during rapid solidification. The specific formula is as follows:

$$
\begin{aligned}
\sigma_{S}=\frac{160 \mu \cdot a}{\left(f_{R}^{S}\right)^{2} t_{f} \lambda_{2}^{2}} \\
\quad \times \frac{\left(1-f_{S}(x)\right)}{f_{S}(x)}\left\{\begin{array}{c}
{\left[\begin{array}{c}
f_{S}(x)-f_{\text {coh }}(x) \\
+\frac{1}{\left(1-f_{S}(x)\right)} \\
-\frac{1}{\left(1-f_{\text {coh }}(x)\right)} \\
+2 \ln \left[\frac{1-f_{S}(x)}{1-f_{\text {coh }}(x)}\right]
\end{array}\right]} \\
+\frac{2\left(1+\beta_{S}\right) \alpha_{\text {ther }} \Delta T_{0} \Delta T}{3 G_{S}} Q\left[f_{S}(x)\right]
\end{array}\right\}
\end{aligned}
$$

where $\mu$ represents the dynamic viscosity, $\alpha$ is the length of the solid-liquid coexistence zone during the recalescence process, $f_{s}(x)$ is the solid volume fraction of primary dendrite, $f_{R}^{S}$ is the solid fraction, $t_{f}$ is the recalescence time, $\lambda_{2}$ is the secondary dendrite arm spacing, $\beta_{s}$ is the solidification shrinkage, $f_{\text {coh }}(x)$ is the solid fraction of the continuous dendrite network, $\alpha_{\text {ther }}$ is the scale factor, $\Delta T$ is undercooling, $\Delta T_{0}$ is the solidification range of the alloy, $G_{S}$ is the temperature gradient at the solidliquid interface in undercooled melt, and $Q\left[f_{s}(x)\right]$ is the volume flow of interdendritic liquid flow. 
(a)
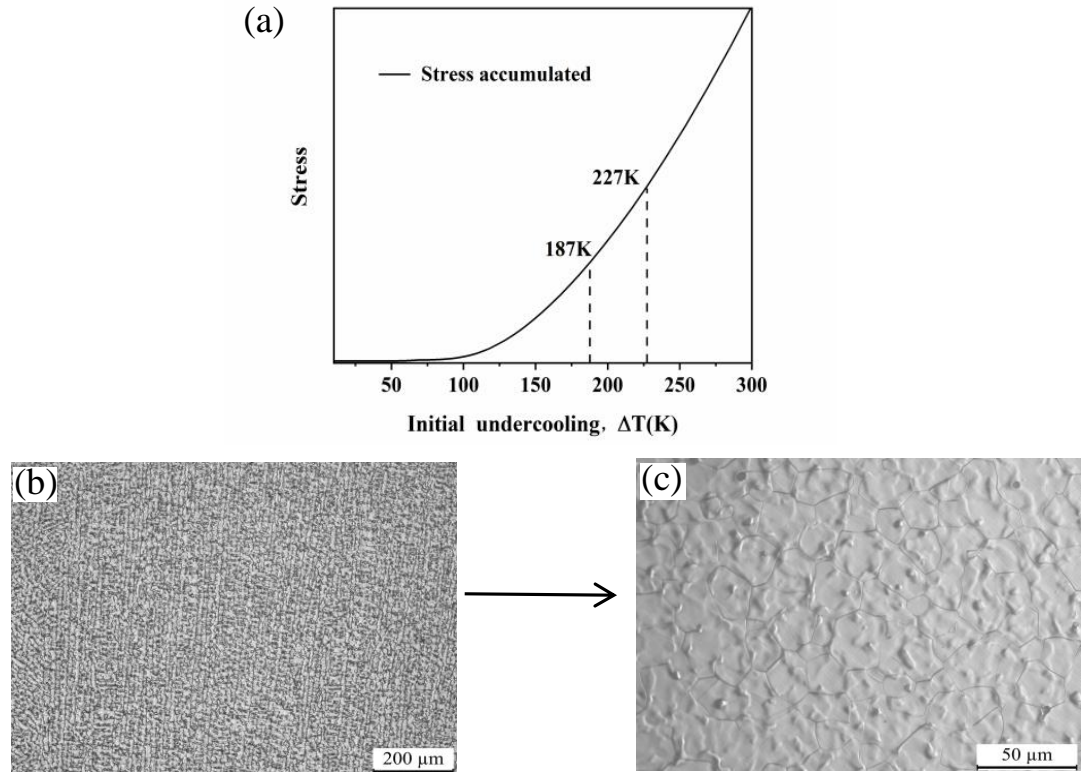

Fig. 7. (A) Relationship between stress accumulation and initial undercooling during rapid solidification of $\mathrm{Cu}_{55} \mathrm{Ni}_{45}$ alloy, (B) Microstructure of $\mathrm{Cu}_{55} \mathrm{Ni}_{45}$ alloy at $\Delta T=187 \mathrm{~K},(\mathbf{C})$ microstructure of $\mathrm{Cu}_{55} \mathrm{Ni}_{45}$ alloy at $\Delta T=227 \mathrm{~K}$.

Based on the above equation, the stress accumulation of undercooled $\mathrm{Cu}_{55} \mathrm{Ni}_{45}$ alloy melt during rapid solidification can be obtained, which is shown in Figure 7 . The diagram can explain the transformation of microstructure from oriented fine dendrites to polygonal equiaxed grains (Figure 7B, 7C). The stress accumulated in the solidified structure of $\mathrm{Cu}_{55} \mathrm{Ni}_{45}$ alloy is very small at low undercooling, but quite large at high undercooling. With the continuous increase of undercooling, the accumulated stress will exceed the strength limit of the alloy, the fine dendrites will break up, and the fine polygonal equiaxed grains will be formed again in the subsequent recrystallization stage.

\subsection{Effect of grain size and microhard- ness on undercooling evolution}

The dendrite and grain sizes were measured by measuring the dendrite trunk length and drawing lines, respectively. Figure 8A shows the evolution relationship between the average grain size and undercooling. All samples were tested on microhardness tester, as shown in Figure 8B, which shows the evolution relationship between microhardness and undercooling.
It is found that the solidification microstructure of $\mathrm{Cu}_{55} \mathrm{Ni}_{45}$ alloy is characterized by directional dendrite with a large undercooling range. In addition, the grain size of the alloy perceptibly decreases twice, and the grain size of the two refinements is also different. The average grain size of the alloy reaches $23 \mu \mathrm{m}$ at the undercooling $\Delta T=70 \mathrm{~K}$, and $11 \mu \mathrm{m}$ at the high undercooling $\Delta T=272 \mathrm{~K}$. The grain size of the grain refinement structure under high undercooling is smaller and more uniform than that under low undercooling. In the undercooling range of 54-96 K, the microhardness of the solidified structure is relatively high, and the trend first increases and then decreases. It is due to the obvious effect of fine grain strengthening, which makes the microstructure hardness higher [28]. With the increase of undercooling, the proportion of fine grains in the structure decreases, and the hardness decreases. In the undercooling range of 96-227 K, the hardness is mainly affected by the accumulation of stress. The accumulated stress in the alloy structure slightly reduces the surface hardness, which is obviously weaker than the fine grain strengthening effect. At the high undercooling, the microhardness of the structure sud- 
(a)

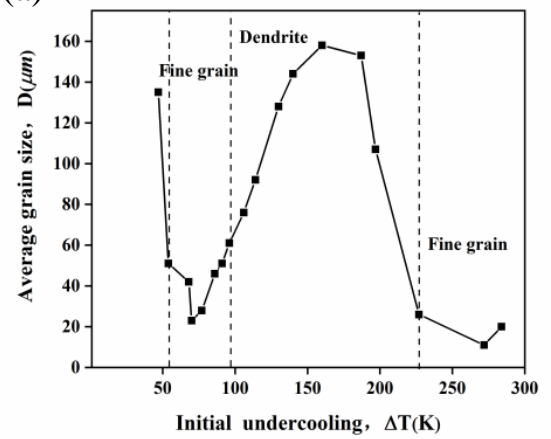

(b)

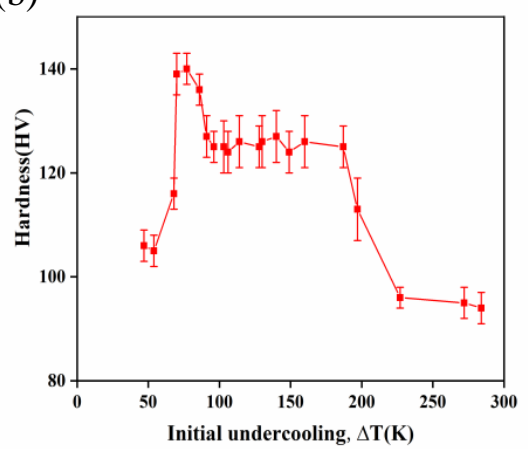

Fig. 8. (A) Evolution of average grain size and undercooling of $\mathrm{Cu}_{55} \mathrm{Ni}_{45}$ alloy, (B) evolution of microhardness and undercooling of $\mathrm{Cu}_{55} \mathrm{Ni}_{45}$ alloy.

denly decreases sharply, which is due to the recrystallization of the structure induced by stress. And the accumulated energy of the system is consumed a lot, resulting in the decrease of hardness. It confirms the mechanism of stress-induced recrystallization of the solidified structure at high undercooling.

\section{Conclusions}

The maximum undercooling of $\mathrm{Cu}_{55} \mathrm{Ni}_{45}$ alloy is ascertained as $284 \mathrm{~K}$ by the combination of molten glass purification and cyclic superheating. Grain refinement occurs twice in the whole undercooling range of the alloy. EBSD technology is used to characterize the grain refinement structure to elaborate the grain refinement mechanism under high undercooling. The main conclusions are as follows:

1. The grain refinement of $\mathrm{Cu}_{55} \mathrm{Ni}_{45}$ alloy occurs at low undercooling and high undercooling. The morphology of grain refinement under low undercooling is spherical equiaxed grains with curved grain boundaries, while the morphology under high undercooling is polygonal grains with flat grain boundaries.

2. Under high undercooling, the grain refinement structure, high proportion of large angle grain boundary and random grain boundary orientation, and a large number of annealing twins indicate that the mi- crostructure recrystallizes under high undercooling, and the microhardness decreases sharply, which also confirms the mechanism of stress-induced recrystallization. In the solidification-recalescence curve, it is found that the maximum recalescence temperature of the low undercooling curve in the rapid solidification stage exceeds the solidus temperature of the alloy, which also explains the dendrite superheating remelting fracture mechanism under low undercooling.

3. The results show that the grain size of the refined structure at high undercooling is smaller than that at low undercooling.

\section{Funding}

This work is supported by the National Natural Science Foundation of China (Nos.51701187, 51804279, 51774254), the Science and Technology Major Project of Shanxi Province (No.MC2016-06), Basic Applied Research Projects in Shanxi Province (201801D221151), and Science and technology innovation project of colleges and universities in Shanxi Province in 2019 (2019L0528).

\section{References}

[1] $\mathrm{Xu} \mathrm{X,} \mathrm{Chen} \mathrm{Y,} \mathrm{Liu} \mathrm{F.} \mathrm{Evidence} \mathrm{of} \mathrm{recrystalliza-}$ tion mechanism of grain refinement in hypercooled Co80Pd20 alloys. Mater Lett. 2012;81:73-5.

[2] Wang H, Liu F, Yang G. Experimental study of grain refinement mechanism in undercooled $\mathrm{Ni}-15 \mathrm{at} . \% \mathrm{Cu}$ alloy. J Mater Res. 2011;25(10):1963-74.

[3] Wang H, An Y, Xu X, Guo X, Hu Y. Rapid solidification microstructure evolution and grain refinement of deeply undercooled nickel alloys. Mater Charact. 2020;170:110703. 
[4] Jie W. Progress of solidification researches and the applications in materials processing. J Mater Sci Technol. 2001;5:575-76.

[5] Xi Z, Yang G, Zhou Y. Growth morphology of Ni3Si in high undercooled Ni-Si eutectic alloy. Prog Nat Sci. 1997;5:114-21.

[6] Liu X, Yang G, Ping F. Solidification behavior of decagonal quasicrystal in the undercooled $\mathrm{Al} 72 \mathrm{Ni} 12$ Co 16 alloy melt. J Mater Sci. 2003;38(5):885-89.

[7] Zhou J, Li J. Effect of denucleating glass composition on undercooling of Fe83Ga17 alloy melts. J Alloys Compd. 2009;467(1-2):179-81.

[8] Perepezko JH. Nucleation reactions in undercooled liquids. Mater Sci Eng A. 1984;178(1):105-111.

[9] Letzig T, Cao CD, Bender W. Dynamics of solidification and development of morphology of Cu-base alloys with a metastable miscibility gap in the range of the undercooled melt. Materialwiss Werkstofftech. 2000;31(9):825-28.

[10] Herlach DM. Non-equilibrium solidification of undercooled metallic metls. Mater Sci Eng R. 1994;12(45):177-272.

[11] Horvay G. The tension field created by a spherical nucleus freezing into its less dense undercooled melt. Int $\mathbf{J}$ Heat Mass Transfer. 1965;8(2):195-243.

[12] Willnecker R, Herlach DM, Feuerbacher B. Grain refinement induced by a critical crystal growth velocity in undercooled melts. Appl Phys Lett. 1990;56(4):324-26.

[13] Jones B, Weston G. Grain refinement in undercooled copper. J Aust Inst Met. 1970;15:3167.

[14] Powell L. The undercooling of silver. J Aust Inst Met. 1965;10:3223.

[15] Karma A. Model of grain refinement in solidification of undercooled melts. Int $\mathrm{J}$ Nonequ Process. 1998;11(2):201-33.

[16] Li J, Liu Y, Lu Y, Yang G, Zhou Y. Structural evolution of undercooled $\mathrm{Ni}-\mathrm{Cu}$ alloys. J Cryst Growth. 1998;192:462-70.

[17] Xu X, Liu F. Recrystallization and twinning in rapidly solidified nickel based alloys without man-made plastic deformation. J Alloys Compd. 2014;615:156-62.
[18] Xu X, Hou H, Zhao Y, Liu F. Nonequilibrium solidification, grain refinements, and recrystallization of deeply undercooled Ni-20 At. Pct Cu Alloys: Effects of remelting and stress. Metall Mater Trans A. 2017;48(10):4777-85.

[19] Xu XL, Liu F. Crystal growth due to recrystallization upon annealing rapid solidification microstructures of deeply undercooled single phase alloys quenched before recalescence. Cryst Growth Des. 2014;14(5):21102114.

[20] Liu F, Yang G. Stress-induced recrystallization mechanism for grain refinement in highly undercooled superalloy. J Cryst Growth. 2001;231:295-305.

[21] Boettinger WJ, Coriell SR, Trivedi R. Rapid solidification processing: principles and technologies IV. Baton Rouge: Claitor's Pulishing Division; 1988.

[22] Boettinger WJ, Aziz MJ. Theory for the trapping of disorder and solute in intermetallic phases by rapid solidification. Acta Metall. 1989;37(12):3379-91.

[23] Jou D, Camacho J, Grmela M. On the nonequilibrium thermodynamics of non-Fickian diffusion. Macromolecules. 1991;24(12):3597-602.

[24] Sobolev SL. Influence of local nonequilibrium on the rapid solidification of binary alloys. Tech Phys. 1998;43(3):307-13.

[25] Wei B, Yang G, Zhou Y. High undercooling and rapid solidi- fication of Ni-32.5\% Sn eutectic alloy. Acta Metall Mater. 1991;39:1249.

[26] Li J, Jie W, Yang G. Solidification structure formation in undercooled Fe-Ni alloy. Acta Mater. 2002;50(7):1797807.

[27] An Y, Liang L, Xu X, Zhao Y, Hou H. Effect of bulk undercooling on microstructure transformation mechanism of rapidly solidified nickel alloys. J Mater Res Technol. 2021;11:548-63.

[28] Shin K, Chung D, Lee S. The effect of consolidation temperature on microstructure and mechanical properties in powder metallurgy-processed $2 \mathrm{XXX}$ aluminum alloy composites reinforced with $\mathrm{SiC}$ particulates. Metall Mater Trans A. 1997;28:2625. 